\title{
Data Structure Specifications via Local Equality Axioms
}

\author{
Scott McPeak and George C. Necula \\ University of California, Berkeley \\ \{smcpeak, necula\}@cs.berkeley.edu
}

\begin{abstract}
We describe a program verification methodology for specifying global shape properties of data structures by means of axioms involving predicates on scalar fields, pointer equalities, and pointer disequalities, in the neighborhood of a memory cell. We show that such local invariants are both natural and sufficient for describing a large class of data structures. We describe a complete decision procedure for axioms without disequalities, and practical heuristics for the full language. The procedure has the key advantage that it can be extended easily with reasoning for any decidable theory of scalar fields.
\end{abstract}

\section{Introduction}

This paper explores a program verification strategy where programs are annotated with invariants, and decision procedures are used to prove them. A key element of such an approach is the specification language, which must precisely capture shape and alias information but also be amenable to automatic reasoning. Type systems and alias analyses are often too imprecise. There are very expressive specification languages (e.g., reachability predicates [15], shape types 4) with either negative or unknown decidability results. A few systems such as TVLA [19] and PALE [13] have similar expressivity and effectiveness, but use logics with transitive closure and thus incur additional restrictions.

We propose to use local equality axioms for data structure specification ("LEADS"), such as "for every list node $n$, n.next.prev $=n$ ", which specifies a doubly-linked list. This simple idea generalizes to describe a wide variety of shapes, as subsequent examples will show. And, as each specification constrains only a bounded fragment of the heap around a distinguished element $n$ (unlike with transitive closure), it is fairly easy to reason about.

There are two main contributions of this work. First, we present a methodology for specifying shapes of data structures, using local specifications. The specifications use arbitrary predicates on scalar fields and equality between pointer expressions to constrain the shape of the data structure. We show that such local specifications can express indirectly a number of important global properties.

The second contribution is a decision procedure for a class of local shape specifications as described above. The decision procedure is based on the idea that local shape specifications have the property that any counterexamples are 
also local. This decision procedure is not only simple to implement but fits naturally in a cooperating decision procedure framework that integrates pointershape reasoning with reasoning about scalar values, such as linear arithmetic, or uninterpreted functions.

A related contribution is to the field of automated deduction, for dealing with universally quantified assumptions: the matching problem is that of finding sufficient instantiations of universally quantified facts to prove a goal. Performing too few instantiations endangers completeness while performing too many compromises the performance of the algorithm and often even its termination. For the class of universally quantified axioms that we consider here we show a complete and terminating matching rule. This is a valuable result in a field where heuristics are the norm [14,2].

Our experimental results are encouraging. We show that we can describe the same data structures that are discussed in the PALE publications, with somewhat better performance results; we can also encode some data structures that are not expressible using PALE. We also show that the matching rules are not only complete, but lead to a factor of two improvement in performance over the heuristics used by Simplify [2], a mature automatic theorem prover. Furthermore, unlike matching in Simplify, our algorithm will always terminate.

\section{Methodology Example}

We follow a standard program verification strategy (e.g., 14]), with programmerspecified invariants for each loop and for the function start (precondition) and end (postcondition). We use a symbolic verification condition generator to extract a verification condition for each path connecting two invariants. The emphasis in this paper is on the specification mechanism for the invariants and the decision procedure for proving the resulting verification conditions.

Suppose we wish to verify the procedure in Figure 1 part of a hypothetical process scheduler written in a Java-like language. It has the job of removing a process from the list of runnable processes, in preparation for putting it into the list of sleeping processes. Each list is doubly-linked.

We capture the data structure invariants using the set of axioms in Figure 1 . where the quantifier ranges over list cells. These axioms constitute the data structure invariant, and must hold at the start and end of the function. Axioms A1 and A2 express the local invariant that the next and prev fields are inverses. Axiom A3 says that all the processes in a list have the same state (RUN or SLP), and axiom A4 says that all the runnable processes have non-increasing priorities.

To begin verifying insert, we need a formal precondition:

$$
P R E: \quad \mathrm{x} \neq \mathrm{null} \wedge \mathrm{x} \cdot \mathrm{prev} \neq \mathrm{null} \wedge \mathrm{x} . \text { state }=\mathrm{RUN}
$$

The verification condition generator produces verification conditions, which are implications where the left-hand side consists of the function precondition along with the current path predicates, and the right-hand side is the goal to prove. 

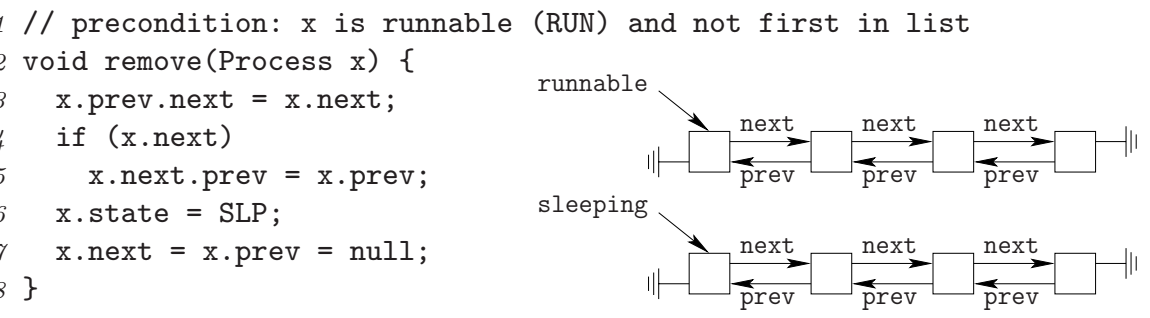

A1. $\forall p . p \neq$ null $\wedge p$.next $\neq$ null

A2. $\forall p . p \neq$ null $\wedge p$.prev $\neq$ null

A3. $\forall p . p \neq$ null $\wedge p$.next $\neq$ null

$\Rightarrow p$.next.prev $=p$

$\Rightarrow p$.prev.next $=p$

$\Rightarrow p$.state $=p$.next.state

A4. $\quad \forall p . p \neq \operatorname{null} \wedge p$.next $\neq$ null $\wedge p$.state $=$ RUN $\Rightarrow p$.prio $\geq p$.next.prio

Fig. 1. A scheduler remove function and its data structure axioms

We use the standard strategy to show validity of the verification condition by showing that its negation is unsatisfiable. For example, to prove that we do not dereference null in $\mathrm{x}$. next on line 3 , we must show unsatisfiability of PRE $\wedge$ $\mathrm{x}=\mathrm{null}$. This can be done without any reference to the axioms.

What is harder to show is that the axioms hold when the function returns. Consider first showing that axiom A3 still holds, which is non-trivial since the axiom depends on the mutated fields next and state. An update $q . f=v$ is modeled by saying that the function modeling field $\mathrm{f}$ is changed into $\mathrm{f}[q \mapsto v]$, with semantics

$$
p . \mathrm{f}[q \mapsto v]= \begin{cases}v & \text { if } p=q \\ p . \mathrm{f} & \text { otherwise }\end{cases}
$$

The updated values of the next and state fields relevant to A3 are:

$$
\begin{aligned}
\text { next } & =\operatorname{next}[\mathrm{x} \cdot \mathrm{prev} \mapsto \mathrm{x} . \mathrm{next}][\mathrm{x} \mapsto \mathrm{null}] \\
\text { state }^{\prime} & =\operatorname{state}[\mathrm{x} \mapsto \mathrm{SLP}]
\end{aligned}
$$

We have to verify that A3 still holds:

$$
\forall q . q \neq \operatorname{null} \wedge q . \text { next' } \neq \text { null } \Rightarrow q . \text { state' }=q \text {.next'.state' } \quad \text { (goal) }
$$

To prove a formula involving updated fields, such as q.next', we first eliminate the field updates by performing the case analysis suggested by the update equation (upd). In each case, what remains to be shown is that a conjunction of literals involving field accesses is unsatisfiable in the presence of some universally quantified local equality axioms.

For our example, there are twelve cases to consider: $q$.next' has cases $q=\mathrm{x}$, $q=\mathrm{x} . p r e v \neq \mathrm{x}$, and $q \neq \mathrm{x} \wedge q \neq \mathrm{x}$.prev; $q$.state' has cases $q=\mathrm{x}$ and $q \neq \mathrm{x}$; and $q$.next'.state' has cases $q$. next' $=\mathrm{x}$ and $q$. next' $\neq \mathrm{x}$. Several cases can be shown unsatisfiable without relying on the axioms.

Four cases require using the axioms; one such case is when $q=\mathrm{x} . \mathrm{prev} \neq \mathrm{x}$ and $q$. next' $=\mathrm{x}$. Consequently $q$.state' $=q$.state, $q$. next' $=$ x.next and 


\begin{tabular}{|c|c|c|}
\hline Injectivity & & $\forall p \cdot p \neq \operatorname{null} \wedge p \cdot \mathbf{a} \neq$ null $\Rightarrow p \cdot \mathbf{a} \cdot \mathbf{b}=p$ \\
\hline Transitivity & & $\forall p \cdot p \neq \operatorname{null} \wedge p \cdot \mathrm{a} \neq \mathrm{null} \Rightarrow p \cdot \mathrm{a} \cdot \mathrm{b}=p \cdot \mathrm{b}$ \\
\hline Order & & $\forall p \cdot p \neq \operatorname{null} \wedge p \cdot \mathrm{a} \neq \operatorname{null} \Rightarrow p \cdot \mathrm{a} \cdot \mathrm{b}>p \cdot \mathrm{b}$ \\
\hline trid & & $\wedge p . \mathrm{a} \neq \operatorname{null} \wedge p . \mathbf{f} \neq \operatorname{null} \Rightarrow p \cdot \mathbf{a} . \mathbf{f}=p . \mathbf{f} . \mathrm{a}$ \\
\hline
\end{tabular}

Fig. 2. Four important axiom forms

$q$. next'.state' $=$ x.next.state. We must show q.state $=$ x.next.state. We first instantiate $A 2$ at $\mathrm{x}$ (written $A 2[\mathrm{x} / p]$ ) to derive x.prev.next $=\mathrm{x}$, implying $q$. next $=\mathrm{x}$. Then we instantiate $A 3[q / p]$ to derive $q$.state $=\mathrm{x}$. state, and finally $A 3[\mathrm{x} / p]$ to derive $\mathrm{x}$. state $=\mathrm{x}$. next.state.

The essence of the above discussion is that the verification strategy will perform case analysis based on the memory writes and will generate facts that must be proved unsatisfiable using a number of axiom instantiations. The only difficulty is how to decide what instances of axioms to use; a strategy that instantiates too few axioms will not be complete (we might fail to prove unsatisfiability), while a strategy that instantiates too many might not terminate.

\subsection{Unrestricted Scalar Predicates}

Scalar predicates let us connect the shape of a data structure with the data stored within it. One advantage of our specification strategy is that we we can combine our satisfiability procedure with that of any predicate that works within the framework of a Nelson-Oppen theorem prover [16]. While other approaches often abstract scalars as boolean fields [13, 19, we can reason about them precisely. For example, in order to verify that the function remove shown in Figure 1 preserves the priority ordering axiom A4, we need transitivity of $\geq$, so we use a satisfiability procedure for partial orders. We also use scalar predicates to allow descriptions for different types of objects to coexist (e.g., a list and a tree), by predicating axioms on the object's dynamic type (modeled as a field).

\subsection{Useful Axiom Patterns}

In the process of specifying data structures, we have identified several very useful axiom patterns; four are shown in Figure 2. For example, axiom A1 of the scheduler example is an instance of the injectivity pattern. Such an injectivity axiom implies useful must-not-alias facts such as $x \neq y \wedge x . \mathrm{a} \neq \mathrm{null} \Rightarrow x . \mathrm{a} \neq y$.a. Injectivity can specify tree shapes as well, for example:

$$
\begin{aligned}
& \forall p . p \neq \operatorname{null} \wedge p . \text { left } \neq \text { null } \Rightarrow p . \text { eft.inv }=p \wedge p . \text { left.kind }=L \\
& \forall p . p \neq \text { null } \wedge p . \text { right } \neq \text { null } \Rightarrow p . \text { right.inv }=p \wedge p . \text { right.kind }=R
\end{aligned}
$$

where kind is a scalar field and $L \neq R$. Such axioms specify that left and right are mutually injective, because inv is the inverse of their union. However, note that (to support data structures with sharing) pointers are not required to be injective; the user states when and where injectivity holds.

The axioms A3 and A4 from the example are similar in that they relate the value of a certain field across the next field, which is in some sense transitive. 
A transitivity axiom can be used to say that two memory cells are in separate instances of a data structure; we used axiom A3 to require that the runnable and the sleeping lists are disjoint. More generally, we can prove the powerful must-not-reach fact $x \cdot \mathrm{b} \neq y \cdot \mathrm{b} \Rightarrow x \cdot \mathrm{a}^{n} \neq y \cdot \mathrm{a}^{m}$, where $x \cdot \mathrm{a}^{n}$ means the object reached from $x$ after following the a field $n$ times. But note that transitivity axioms cannot express must-reach facts.

Axiom A4 is a non-strict order axiom. In its strict form it can be used to specify the absence of cycles. In this pattern, any transitive and anti-reflexive binary predicate can be used in place of $>$.

We defer discussion of the grid pattern to Section 11. A common theme in these patterns is that we use equalities and scalar predicates to imply disequalities, which are needed to reason precisely about updates. The interplay between equality axioms and the entailed disequalities is central to our approach.

\subsection{Ghost Fields}

Often, a data structure does not physically have a field that is needed to specify its shape. For example, a singly-linked list does not have the back pointers needed to witness injectivity of the forward pointers. In such cases, we propose to simply add ghost fields (also known as auxiliary variables, or specification fields), which are fields that our verification algorithm treats the same as "real" fields, but do not actually exist when the code is compiled and run. Thus, to specify a singlylinked list, we add prev as a ghost field and instead specify a doubly-linked list. For our prototype tool, the programmer must add explicit updates to ghost fields. Updates to ghost fields often follow a regular pattern, so are presumably amenable to inference, but in the limit human assistance is required [10].

\subsection{Temporary Invariant Breakage}

In our tool, the shape descriptions are required to accurately describe the heap at procedure entry and exit, and at all loop invariant points. But some programs need their invariants to be broken temporarily at such points. Our solution is to introduce a special ghost global pointer called (say) broken, and write axioms of the form

$$
\forall p . \quad p \neq \text { broken } \Rightarrow Q(p)
$$

where $Q(p)$ is the nominal invariant. Then the program can set broken to point at whichever object (if any) does not respect the invariant. Once the object's invariant is repaired, broken can be set to null, meaning all invariants hold.

\section{The Specification Language}

Figure 3describes the main syntactic elements of the specification language. This is a two-sorted logic, with pointer values and scalar values. Scalar predicates may have any arity. We use the notation $\mathcal{E}$ for disjunctions of pointer equalities, $\mathcal{D}$ for disjunctions of pointer disequalities, and $\mathcal{C}$ for disjunctions of scalar constraints. 


\begin{tabular}{|c|c|c|c|c|c|c|c|}
\hline Globals & $x$ & $\epsilon$ & Var & Pointer equalities & $E$ & $::=$ & $t_{1}=t_{2}$ \\
\hline Variables & $p$ & $\in$ & Var & Pointer disequal. & $D$ & $::=$ & $t_{1} \neq t_{2}$ \\
\hline Pointer fields & $L$ & $\epsilon$ & PField & Scalar fields & $S, R$ & $\in$ & SField \\
\hline Pointer paths & $\alpha, \beta$ & $::=$ & $\epsilon \mid \alpha . L$ & Scalar predicates & $P$ & $\epsilon$ & Pred \\
\hline Pointer terms & $t, u$ & $::=$ & null $\mid x$ & Scalar constraints & $C$ & $::=$ & $P\left(t_{1} . S, t_{2} \cdot R\right)$ \\
\hline
\end{tabular}

Fig. 3. Specification language

Core Language. We have two languages, one a subset of the other. In the core language, a data structure specification is a finite set of axioms of the form

$$
\forall p . \quad \mathcal{E} \vee \mathcal{C}
$$

where $p$ ranges over pointer values. Axioms in the core language cannot have pointer disequalities, but can have scalar field disequalities in $\mathcal{C}$. This language is expressive enough to describe many common structures, including those of Figures 1] and 2] Section 4 describes a satisfiability procedure for this language.

Extended Language. The extended language has axioms of the form

$$
\forall p . \quad \mathcal{E} \vee \mathcal{C} \vee \mathcal{D}
$$

This language is more expressive; e.g., it allows us to insist that certain pointers not be null, or to describe additional kinds of reachability, or to require that a structure be cyclic, among other things. Unfortunately, the extended language includes specifications with undecidable theories (see below). However, we extend the satisfiability procedure for the core language to handle many extended axioms as well, including all forms that we have encountered in our experiments.

Nullable Subterms. Data structure specification axioms naturally have the following nullable subterms (NS) property: for any pointer term t.L or any scalar term $t . S$ that appears in the body of an axiom, the axiom also contains the equality $t=$ null among its disjuncts. This is because fields are not defined at null. We require that all axioms have the NS property.

Discussion. The keystone of our technical result is the observation that the NS property ensures the decidability of the axioms in the core language. In contrast, if we allow axioms of the form $\forall p . p . \alpha=p \cdot \beta$ (in the core language, but without the NS property), then we could encode any instance of the (undecidable) "word problem" 8] as an axiom set and a satisfiability query.

Intuitively, an axiom with the nullable subterms property can be satisfied by setting to null any unconstrained subterms. This avoids having to materialize new terms, which in turn ensures termination. Notice however, that if we allow arbitrary pointer disequalities we can cancel the effect of the NS condition. For example, the axiom

$$
\forall p . \quad p=\operatorname{null} \vee p . \mathrm{a} \neq \mathrm{null}
$$


forces t.a to be non-null for any non-null $t$. Thus the unrestricted use of the disequalities in $\mathcal{D}$ makes satisfiability undecidable. In our experiments we observed that pointer disequalities are needed less frequently than other forms, which motivates separate treatment of the core and extended languages.

\section{A Satisfiability Algorithm}

The algorithm is essentially a Nelson-Oppen theorem prover, augmented with specific matching rules for instantiating the quantified axioms, and deferred, heuristic treatment of disequalities among uninstantiated terms.

\subsection{The Algorithm}

The purpose of the algorithm is to determine whether a set of local equality axioms and a set of ground (unquantified) facts is satisfiable. When used on axioms without pointer disequalities (core language) the algorithm always terminates with a definite answer. However, in the presence of axioms with pointer disequalities (extended language) the algorithm may return "maybe satisfiable". Note that pointer disequalities in the ground facts do not endanger completeness.

The basic idea of the algorithm is to exploit the observation that in a data structure described by local axioms, when the facts are satisfiable, they are satisfiable by a "small" model. Essentially, the algorithm attempts to construct such a model by setting any unknown values to null.

The algorithm's central data structure is an equality graph (e-graph) $G$ [16], a congruence-closed representation of the ground facts. We use the notation $t \in G$ to mean term $t$ is represented in $G$, and $G \wedge f$ to mean the e-graph obtained by adding representatives of the terms in formula $f$ to $G$, and asserting $f$.

The algorithm must decide which axioms to instantiate and for which terms. We first define when a ground pointer term $u$ matches an equality disjunct $t_{1}=t_{2}$ in an axiom with bound (quantified) variable $p$, as follows:

- Either $t_{1}[u / p] \in G$ or $t_{2}[u / p] \in G$, when neither $t_{1}$ nor $t_{2}$ is null, or

- $t_{1}$ is null and $t_{2}[u / p] \in G$ (or vice-versa).

We say that a term matches an axiom if it matches all its pointer equality disjuncts. An axiom is instantiated with any term that matches the axiom.

These rules implement an "all/most" strategy of instantiation. For example, an axiom

$$
\forall p . \quad \ldots \vee p . \alpha . \mathrm{a}=p . \beta . \mathrm{b}
$$

must include (because of NS) disjuncts $p . \alpha=$ null and $p . \beta=$ null. For a ground term $u$ to match this axiom, it must match every equality disjunct (from the definition above), so $u . \alpha, u . \beta$, and either $u . \alpha . \mathrm{a}$ or $u . \beta$.b must be represented. Consequently, asserting the literal $u . \alpha . \mathrm{a}=u . \beta . \mathrm{b}$ will require representing at most one new term, but no new equivalence classes.

If $G$ is an e-graph, and $D S$ is a conjunction (i.e., a set) of disjunctions of pointer disequalities, we define a satisfiability procedure unsat $(G, D S)$ that returns true if the facts represented in $G$ along with $D S$ and the axioms are 


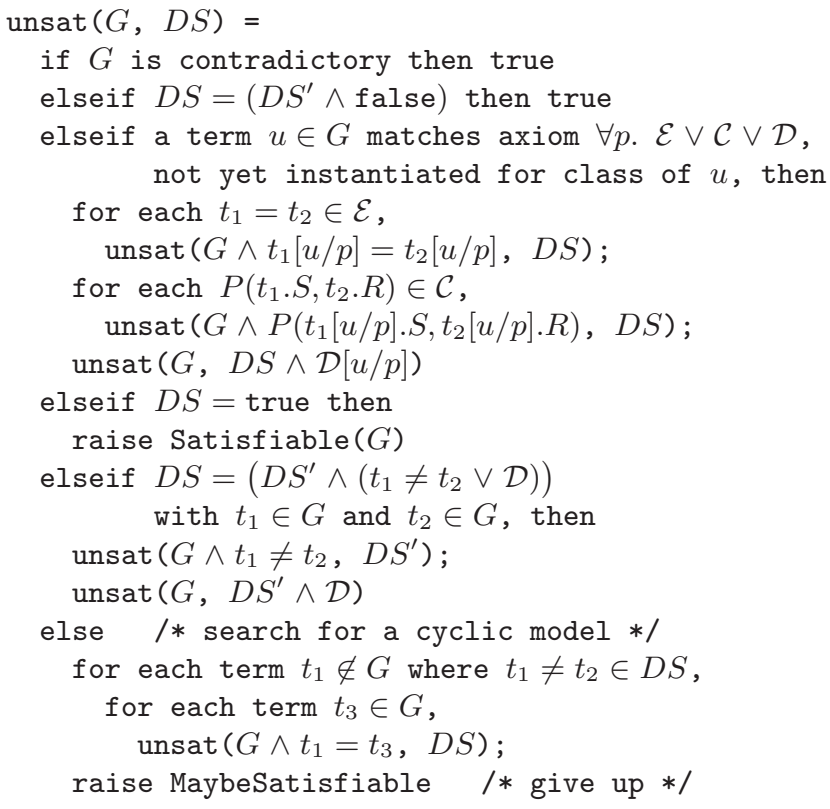

Fig. 4. The basic decision algorithm

unsatisfiable, raises the exception Satisfiable if it is satisfiable, and raises the exception MaybeSatisfiable if the procedure cannot decide satisfiability (in presence of axioms with pointer disequalities). Figure 4 contains the pseudocode for this procedure. This procedure is used by first representing the facts in an e-graph $G$, and then invoking unsat $(G$, true).

Lines 2 and 3 identify contradictions in the e-graph. The judgment " $G$ is contradictory" may make use of a separate satisfiability procedure for the scalar predicates. We write $D S=\left(D S^{\prime} \wedge\right.$ false $)$ to deconstruct $D S$. The heart of the algorithm is in lines $4-10$, which instantiate axioms with terms that match, given the matching rules described above, and performs the case analysis dictated by the instantiated axioms. Note that the pointer disequalities are deferred by collecting them in $D S$ (line 10). When line 12 is reached, the axioms are completely instantiated and all cases fully analyzed, but no contradiction has been found. Thus, the original $G \wedge D S$ was satisfiable; the current $G$ is a witness.

Lines 13-16 handle pointer disequalities where both sides are already represented in the e-graph. Finally, lines 17-20 attempt to find a satisfying assignment for the unrepresented terms in $D S$ by setting them equal to other terms that are represented. The algorithm searches for cyclic models to accommodate data structures like cyclic lists.

Recalling the proof from Section 2 that A3 holds after next and state have been modified, we can now explain how the algorithm would prove it. First, the update rules are used to split the proof into cases; among those cases is $q \neq \mathrm{x} \wedge q=\mathrm{x}$. prev $\wedge \mathrm{x}$.next $\neq \mathrm{x}$, which (along with the precondition) is used 
to initialize $G$, and unsat $(G$, true) is invoked. Next, the algorithm determines that the term $\mathrm{x}$ matches axiom $A 2$, because x.prev $\in G$, and begins asserting its disjuncts. Only the x.prev.next $=\mathrm{x}$ disjunct is not immediately refuted. Asserting this literal causes the new term x.prev.next to be represented, by putting it into the same equivalence class as $\mathrm{x}$. Since $q=\mathrm{x}$.prev, the term $q$.next is now represented, and so $q$ matches $A 3$, and $A 3[q / p]$ is instantiated. Finally, as x.next is represented, $A 3[\mathrm{x} / p]$ is also instantiated. After these three instantiations, case analysis and reasoning about equality are sufficient.

\subsection{Analysis of the Algorithm}

Correctness when result is "Unsatisfiable". The algorithm is always right when it claims unsatisfiability, which in our methodology means that the goal is proved. The (omitted) proof is a straightforward induction on the recursion.

Correctness when result is "Satisfiable". When the algorithm raises Satisfiable $\left(G^{\prime}\right)$, there is a model $\Psi$ that satisfies the original $G \wedge D S \wedge \mathcal{A}$, where $\mathcal{A}$ is the set of axioms:

$$
\Psi(u)= \begin{cases}u^{*} & \text { if } u \in G^{\prime} \\ \text { null } & \text { otherwise }\end{cases}
$$

where by $u^{*}$ we denote the representative of $u$ in the e-graph $G^{\prime} . \Psi$ satisfies $\mathcal{A}$ because for every pointer term $u$ (represented or not) and axiom $A$, either $A[u / p]$ has been instantiated, so $G^{\prime}$ satisfies $A[u / p]$ (and so does $\Psi$ ), or else $u$ does not match $A$, in which case there is a pointer equality $t_{1}=t_{2}$ in $A$ that does not match. By the definition of matching, there are two cases:

- Neither $t_{1}$ nor $t_{2}$ are null, and $t_{1}[u / p] \notin G$ and $t_{2}[u / p] \notin G$. But then $\Psi\left(t_{1}[u / p]\right)=\Psi\left(t_{2}[u / p]\right)=$ null, satisfying $A[u / p]$.

- $t_{1}$ is null and $t_{2}[u / p] \notin G$. Then $\Psi\left(t_{2}[u / p]\right)=$ null, satisfying $A[u / p]$.

That is, the matching rules guarantee that if an axiom has not been instantiated, it can be satisfied by setting unrepresented terms to null.

Termination. The essence of the termination argument is that none of the steps of the algorithm increases the number of pointer equivalence classes in the e-graph. Whenever a new pointer term $t$ might be created in the process of extending the e-graph, it is created while adding the fact $t=t^{\prime}$, where $t^{\prime}$ is already in the e-graph. This is ensured by the matching rule that requires one side of each equality to be present in the e-graph. Furthermore, if $t$ is of the form $t^{\prime \prime} . L$, then by the NS property, the disjunct $t^{\prime \prime}=$ null is also part of axiom and thus the matching rule requires $t^{\prime \prime}$ to be already in the e-graph. Thus $t$ is the only new term and is added to an existing equivalence class. There are only a finite number of equality and scalar constraints over a fixed number of pointer equivalence classes; once all entailed constraints are found, the algorithm halts. 
Soundness and completeness on the Core Language. For the core language, the algorithm stays above line 13 , because $D S$ is always empty (true). As it always terminates, it must always return either Satisfiable or Unsatisfiable.

Complexity. The algorithm has exponential complexity, because of the case analysis that is performed in processing updates and conditional axioms. If we have $n$ nodes in the e-graph representing the facts, $a$ axioms with at most $k$ disjuncts each, then there could be a total of $n \times a$ axiom instantiations. We can think of the algorithm exploring a state space that resembles a tree. Each node in the tree corresponds to an instance of an axiom, and has a branching factor $k$. Consequently its running time is $O\left(k^{n a} S(n)\right)$, where $S(n)$ is the complexity bound for the underlying satisfiability procedure for scalar constraints.

\section{Disequalities}

Axioms with disequalities are necessary for specifying some shape properties, such as never-null pointers and cyclic structures. These often take the form of termination conditions, for example

$$
\forall p . Q(p) \Rightarrow p \text {.next } \neq \text { null }
$$

This poses a challenge for automated reasoning, as the basic approach is to search for a finite model, so we try to set pointers to null wherever possible, but setting $p$.next to null is impossible if $Q(p)$ is known to be true.

In the context of the algorithm in Figure 4, this issue arises when we reach line 17: all axioms have been instantiated, and $G$ is consistent, but there remain some disequalities $D S$ among terms not represented in $G$. We cannot report satisfiability because the consequences of $D S$ have not yet been considered.

One approach, shown in Figure 4 , is to explicitly check for a cyclic model: try setting unrepresented terms equal to existing terms. When the axioms describe a cyclic structure, this may be the only way to find a finite model.

Another approach (not shown in the figure for reasons of space) is to simply grow the model, representing the terms in $D S$ by creating new equivalence classes. Of course, doing so jeopardizes termination, since the new equivalence classes may trigger additional axiom instantiations. So, the programmer may specify an expansion horizon $k$, and the algorithm will then not create an equivalence class more than $k$ "hops" away from the original set of classes. Typically, we use the bound $k=2$.

If the heuristics fail, the algorithm may report MaybeSatisfiable; but the algorithm will never make a false claim (in particular, the expansion bound is not involved in a claim of unsatisfiability).

More generally (and optimistically), it is possible to show for specific axiom sets that the above heuristics are complete: that the algorithm will never report MaybeSatisfiable. However, it remains future work to develop a systematic way of constructing completeness arguments for axiom sets containing disequalities. 


\section{Experiments}

Our algorithm has been implemented as a modified version of the Simplify [2] theorem prover. To evaluate the expressiveness of our specification language, we compare our prototype verifier to the Pointer Assertion Logic Engine (PALE) [13. To measure the effectiveness of our decision algorithm, we compare it to the unmodified Simplify. For several example programs, we measure the lines of annotation, which axiom forms are used (from Figure 2, plus disequalities), and the time to verify on a $1.1 \mathrm{GHz}$ Athlon Linux PC with 768MB RAM. See Figure 5.

\begin{tabular}{|c|c|c|c|c|c|c|c|c|c|}
\hline \multirow{2}{*}{$\begin{array}{l}\text { example } \\
\text { bubblesort }\end{array}$} & \multirow{2}{*}{$\begin{array}{r}\mathrm{LOC} \\
47\end{array}$} & \multicolumn{2}{|c|}{ Annot. lines } & \multicolumn{3}{|c|}{ Axiom Forms } & \multicolumn{3}{|c|}{ Verify Time (s) } \\
\hline & & 10 & 31 & 2 & & & 1.9 & 0.9 & 0.6 \\
\hline doublylinked & 73 & 35 & 69 & 4 & 1 & & 2.6 & 2.9 & 1.9 \\
\hline taillist & 73 & 38 & 94 & 3 & 4 & & 2.4 & 1.8 & 1.3 \\
\hline redblacktree & 146 & 112 & 224 & 4 & & 4 & 19.4 & 36.5 & 22.0 \\
\hline gc_copy & 38 & & 58 & $\begin{array}{ll}1 & 1\end{array}$ & & 2 & & 50.2 & 15.6 \\
\hline btree & 163 & & 185 & 27 & 4 & 6 & & 96.7 & 26.4 \\
\hline set_as_list & 36 & & 73 & 12 & 1 & 4 & & 1.9 & 1.3 \\
\hline pc_keyb & 1116 & & 163 & 2 & & & & 40.1 & 38.1 \\
\hline scull & 534 & & 360 & 45 & & & & 58.2 & 46.4 \\
\hline
\end{tabular}

Fig. 5. Programs verified, with comparison to PALE when available

We are able to verify all of the examples distributed with PALE1, however PALE requires less than half the lines of annotation. There are three reasons: (1) in PALE, the backbone tree is concisely specified by the data keyword, whereas our axioms must spell it out with injectivity, (2) PALE specifications use transitive closure, whereas we use the more verbose transitivity axioms, and (3) our language does not assume the program is type-safe (it is C, after all), so we have explicit axioms to declare where typing invariants hold. We plan to add forms of syntactic sugar to address these points.

We also selected two data structures that cannot be specified in PALE. gc_copy is the copy phase of a copying garbage collector, and has the job of building an isomorphic copy of the from-space, as shown at right. This isomorphism, embodied by the forwarding pointers $f$, is specified by the "grid" or "homomorphism" axiom $\forall p . \quad \ldots \Rightarrow p$. a.f = p.f.a.

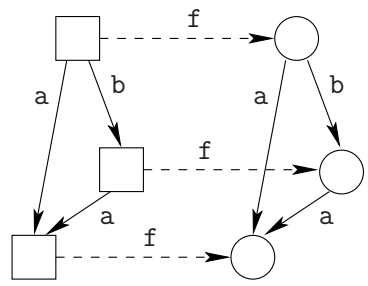
This axiom (along with injectivity of $f$ ) describes a data structure where the targets of $f$ pointers are isomorphic images of the sources.

\footnotetext{
${ }^{1}$ In the case of the taillist example, the verified property is slightly weaker due to the encoding of reachability via transitivity.
} 
The btree benchmark is a B+-tree implementation, interesting in part because we can verify that the tree is balanced, by using scalar constraints

$$
\begin{aligned}
& \forall p . \quad p \neq \text { null } \wedge p . \text { left } \neq \text { null } \Rightarrow p . \text { level }=p . \text { left.level }+1 \\
& \forall p . \quad p \neq \text { null } \wedge p . \text { right } \neq \text { null } \Rightarrow p . \text { level }=p . \text { right.level }+1
\end{aligned}
$$

along with disequality constraints that force all leaves to have the same level:

$$
\begin{array}{ll}
\forall p . & p \neq \text { null } \wedge p . \text { left }=\text { null } \Rightarrow p . \text { level }=0 \\
\forall p . & p \neq \text { null } \wedge p . \text { right }=\text { null } \Rightarrow p . \text { level }=0
\end{array}
$$

However, this specification is also noteworthy because there is no bound $k$ on the size of models as advocated in Section 5 the facts $x .1$ evel $=100$ and $x \neq$ null (plus the axioms) are satisfiable only by models with at least 100 elements. Fortunately, such pathological hypotheses do not seem to arise in practice.

The set_as_list example uses a linked list to represent a set. We use a ghost field $\mathbf{s}$, associated with each node in the list, with intended invariant

$$
\forall n . n . \mathbf{s}=\left\{x \mid \exists i \in \mathbb{N} . n(\text { next })^{i} . \operatorname{data}=x\right\}
$$

That is, s contains all data elements at or below the node. But since our language does not allow " $n(\text {.next })^{i}$ ", we instead write

$$
\begin{aligned}
& \text { S1. } \quad \forall n . \quad n . \text { next } \neq \text { null } \Rightarrow n . s=\{n . \text { data }\} \cup n . \text { next.s } \\
& \text { S2. } \quad \forall n . \quad n . \text { next }=\text { null } \Rightarrow n . s=\{n . \text { data }\}
\end{aligned}
$$

Axiom S1 imposes a lower bound on s, allowing one to conclude (among other things) must-not-reach facts. Axiom S2 imposes an upper bound, and allows conclusion of must-reach facts, but includes a pointer disequality disjunct. To reason about set-theoretic concepts, we use the procedure in 11. This example highlights the way our technique can integrate with powerful off-the-shelf "scalar" concepts to specify the relationship between shape and data.

Finally, pc_keyb and scull are two Linux device drivers. These real-world examples demonstrate the applicability of the technique, and the benefits of integrated pointer and scalar reasoning; for example, their data structures feature arrays of pointers, which are difficult to model in PALE or TVLA.

An important contribution of this work is the particular matching rules used to instantiate the axioms. Simplify has heuristics for creating matching rules for arbitrary universally-quantified facts. As expected, we find that our matching rules, which instantiate the axioms in strictly fewer circumstances, lead to better performance (compare timing columns "Simplify" and "Ours" in Figure 5). In fact, Simplify's heuristics will often lead to infinite matching loops, especially while trying to find counterexamples for invalid goals. This is not to denounce Simplify's heuristics - they do a good job for a wide variety of axiom sets - just to emphasize that in the case of axioms expressing data structure shape within our methodology, one can have a more efficient and predictable algorithm. 


\section{$7 \quad$ Related Work}

As explained in Section 1 most existing approaches to specifying shape are either too imprecise or too difficult to reason about automatically. Here, we consider alternative approaches with similar expressiveness and effectiveness.

PALE 13, the Pointer Assertion Logic Engine, uses graph types [7] to specify a data structure as consisting of a spanning tree backbone augmented with auxiliary pointers. One disadvantage is the restriction that the data structure have a tree backbone: disequality constraints that force certain pointers to not be null are not possible, since every tree pointer is implicitly nullable, cyclic structures are awkward, and grid structures such as the garbage collector example in Section 1 are impossible. The second disadvantage is that only boolean scalar fields are allowed. Thus, all scalar values must first be abstracted into a set of boolean ghost fields, and updates inserted accordingly.

TVLA 12, 19, the Three Valued Logic Analyzer, uses abstract interpretation over a heap description that includes $1 / 2$ or "don't know" values. It obtains shape precision through the use of instrumentation predicates, which are essentially ghost boolean fields with values defined by logical formulas. In general, the programmer specifies how instrumentation predicates evolve across updates, though TVLA can conservatively infer update rules that are often sufficiently precise. The primary advantage of TVLA is it infers loop invariants automatically, by fixpoint iteration. The disadvantage is that the obligation of proving that a shape is preserved across updates is delegated to the instrumentation predicate evolution rules, which are not fully automated. Also, as with PALE, scalar values must be abstracted as boolean instrumentation predicates.

PALE and TVLA include transitive closure operators, and hence can reason directly about reachability, but they pay a price: PALE has non-elementary complexity and requires tree backbones, and TVLA has difficulty evolving instrumentation predicates when they use transitive closure. The difficulties of reasoning about transitive closure have been recently explored by Immerman et. al [6], with significant negative decidability results. Our technique is to approximate reachability using transitivity axioms, giving up some shape precision in exchange for more precision with respect to scalar values.

The shape analysis algorithm of Hackett and Rugina [5] partitions the heap into regions and infers points-to relationships among them. Its shape descriptions are less precise; it can describe singly-linked lists but not doubly-linked lists.

Roles 9] characterize an object by the types it points to, and the types that point at it. Role specifications are similar to our injectivity axioms. The role analysis in [9] provides greater automation but it can express fewer shapes.

Separation logic [18] includes a notion of temporal locality, exploited by a frame rule that allows reasoning about only those heap areas accessed by a procedure. We believe such a notion is essentially orthogonal to, and could be useful with, the spatial locality notions of this paper.

Early work on data structures showed that the property of an object being uniquely generated (e.g., every instance of cons $(1,2)$ is the same object) has decidable consequences [17], a result related to the decidability of the consequences 
of the injectivity axioms presented here. However, the early work does not admit more refined notions of shape, as it does not address the use of quantifiers.

Our work uses methods most similar to the Extended Static Checker 3 and Boogie/Spec\# [1]. However, while we suspect that specifications similar to elements described here have been written before while verifying programs, we are unaware of any attempts to explore their expressiveness or decidability.

\section{Conclusion}

We have presented a language for describing data structure shapes along with scalar field relationships. The language is relatively simple because it can only talk about local properties of a neighborhood of nodes, yet is capable of expressing a wide range of global shape constraints sufficient to imply the kind of must-not-alias information needed for strong update reasoning. Furthermore, it is sufficiently tractable to admit a simple decision procedure, extensible with arbitrary decidable scalar predicates. Using this language we have verified a number of small example programs, of both theoretical and practical interest.

\section{References}

1. D. Cantone and C. G. Zarba. A new fast decision procedure for an unquantified fragment of set theory. In First-Order Theorem Proving, pages 97-105, 1998.

2. D. Detlefs, G. Nelson, and J. B. Saxe. Simplify: A theorem prover for program checking. Technical Report HPL-2003-148, HP Labs, July 2003.

3. D. L. Detlefs, K. R. M. Leino, G. Nelson, and J. B. Saxe. Extended static checking. Technical Report SRC-159, COMPAQ SRC, Palo Alto, USA, Dec. 1998.

4. P. Fradet and D. L. Métayer. Shape types. In POPL, pages 27-39, 1997.

5. B. Hackett and R. Rugina. Region-based shape analysis with tracked locations. In POPL, pages 310-323, 2005.

6. N. Immerman, A. Rabinovich, T. Reps, M. Sagiv, and G. Yorsh. The boundary between decidability and undecidability for transitive-closure logics. In CSL, 2004.

7. N. Klarlund and M. Schwartzbach. Graph types. In POPL, pages 196-205, 1993.

8. D. E. Knuth and P. B. Bendix. Simple word problems in universal algebra. In J. Leech, editor, Computational Problems in Abstract Algebras, pages 263-297. Pergamon Press, 1970.

9. V. Kuncak, P. Lam, and M. Rinard. Role analysis. In POPL, pages 17-32, 2002.

10. V. Kuncak and M. Rinard. Existential heap abstraction entailment is undecidable. In $S A S$, pages 418-438, 2003.

11. K. R. M. Leino and P. Müller. Object invariants in dynamic contexts. In European Conference on Object-Oriented Programming (ECOOP), 2004.

12. T. Lev-Ami and S. Sagiv. TVLA: A system for implementing static analyses. In Static Analysis Symposium, pages 280-301, 2000.

13. A. Möller and M. Schwartzbach. The pointer assertion logic engine. In PLDI, pages 221-231, 2001.

14. G. Nelson. Techniques for program verification. Technical Report CSL-81-10, Xerox Palo Alto Research Center, 1981.

15. G. Nelson. Verifying reachability invariants of linked structures. In $P O P L$, pages 38-47, 1983. 
16. G. Nelson and D. C. Oppen. Fast decision procedures based on congruence closure. JACM, 27(2):356-364, Apr. 1980.

17. D. C. Oppen. Reasoning about recursively defined data structures. JACM, 27(3):403-411, July 1980.

18. J. C. Reynolds. Separation logic: A logic for shared mutable data structures. In Logic in Computer Science, pages 55-74, 2002.

19. S. Sagiv, T. W. Reps, and R. Wilhelm. Parametric shape analysis via 3 -valued logic. TOPLAS, 24(3):217-298, 2002. 